RAPID COMMUNICATION

\title{
Implantation of platelet-rich fibrin and cartilage granules facilitates cartilage repair in the injured rabbit knee: preliminary report
}

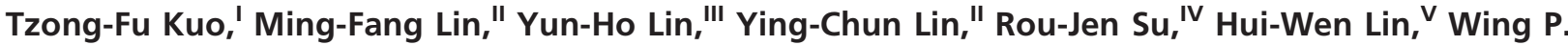 \\ Chan ${ }^{\text {IIIV* }}$
}

'Graduate Institute of Veterinary Medicine, National Taiwan University, Taiwan, Republic of China. "Department of Radiology, Wan Fang Hospital, Taipei Medical University, Taiwan, Republic of China. "'Department of Pathology, School of Medicine, Taipei Medical University, Taiwan, Republic of China.

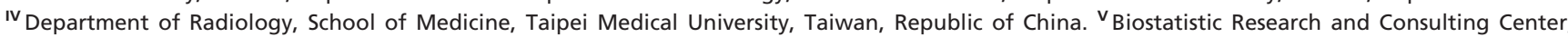
Taipei Medical University, Taiwan, Republic of China.

Email: wingchan@tmu.edu.tw

Tel.: 8862 2930-7930 Ext. 1300

*Contact author: Wing P. Chan

\section{INTRODUCTION}

Autologous chondrocyte implantation has become the current principal procedure for cell-based cartilage repair. ${ }^{1,2}$ However, the disadvantages of autologous chondrocyte implantation include the necessity to perform two surgical procedures: isolation of the chondrocytes from a tissue sample that has been extracted from the patient, which is time-consuming, and implantation of the harvested chondrocytes after expansion in vitro, ${ }^{3}$ which has the potential to induce chondrocyte leakage. ${ }^{4}$

Platelet-rich fibrin (PRF) is a new generation of platelet concentrate that, without anticoagulant, activates the platelets of a blood sample and induces degranulation and cytokine release. ${ }^{5}$ The preparation of PRF is simple and can be completed during one procedure. In dental procedures, when a cystic cavity is filled with PRF, the physiological healing period can be reduced to two months instead of the usual 6 to 12 months. ${ }^{6}$

The aim of this study was to investigate the regeneration of articular cartilage defects of the knee following the implantation of PRF with cartilage granules in a one-step procedure in rabbits using T2-map magnetic resonance imaging (MRI) and histology to assess the rabbits' response to the procedure.

\section{MATERIALS AND METHODS}

The Institutional Animal Care and Use Committee at the National Taiwan University and Taipei Medical University approved the breeding of the animals and the protocol. A total of 12 New Zealand white rabbits (age: 10-12 weeks; weight: $2-3 \mathrm{~kg}$ ) were divided into two groups. In group A $(n=6)$, a cartilage defect was created with no implantation. In group B $(n=6)$, a cartilage defect was created, and a mixture of PRF and cartilage granules was implanted. In

Copyright (c) 2011 CLINICS - This is an Open Access article distributed unde the terms of the Creative Commons Attribution Non-Commercial License (http:// creativecommons.org/licenses/by-nc/3.0/) which permits unrestricted noncommercial use, distribution, and reproduction in any medium, provided the original work is properly cited.

No potential conflict of interest was reported. group B, one rabbit's unoperated left hind leg was used as a positive control and as a tissue morphological reference for histology.

The PRF was prepared using the technique described by Dohan et al. ${ }^{7}$ Immediately after $3 \mathrm{cc}$ of blood had been drawn from the jugular vein of each rabbit during surgery, the blood sample was centrifuged in a 5-mL sterile tube (without anticoagulant) at $400 \mathrm{~g}$ for $10 \mathrm{~min}$ using a PC-02 laboratory centrifuge (Process, Nice, France). Using forceps, the PRF gel (Figure 1) was removed from the middle layer of the sample in the tube; this layer was located between the red corpuscles at the bottom of the tube and acellular plasma at the top of the tube. The total volume of PRF was calculated to be $0.77 \mathrm{~cm}^{3}$.

The PRF gel was sectioned into two to three pieces, mixed with the cartilage fragment that was obtained from the created cartilage defect and ground into small granules (approximately $1 \mathrm{~mm}$ in diameter) to fill in the excision defect.

Surgery was performed under general anesthesia by a senior surgeon (T-F. K) using magnifying loupes $(2.5 \times$, Heine HR, Germany). The rabbits were injected intramuscularly with a mixture of ketamine $(100 \mathrm{mg} / \mathrm{mL}, 0.6 \mathrm{~mL} / \mathrm{kg}$ body weight) and xylazine $(20 \mathrm{mg} / \mathrm{mL}, 0.3 \mathrm{~mL} / \mathrm{kg}$ body weight). In each animal, a hollow cylindrical scoring device with a graduated diameter was used to create a cartilage defect surgically (diameter: $3 \mathrm{~mm}$; depth: $0.5 \mathrm{~mm}$ ) in the weight-bearing portion of the medial femoral condyle of the right hind leg knee. In both groups, the holes were covered with a periosteal patch and secured with sutures. The periosteal patch that corresponded to the size of the area of cartilage damage had been harvested from the tibial shaft. Suturing was started from the medial side using a fourcorner technique with a 7-0 absorbable suture (Prolene, Ethicon, Johnson \& Johnson). The area between the 9 o'clock and 3 o'clock positions was left unsutured for the purpose of PRF and cartilage implantation. Once the implantation was performed, the suturing was completed.

Three months after surgery, the animals were scanned using MRI, which was performed using a 1.5-T magnet (Signa Horizon LX, GE Medical Systems, Milwaukee, WI, USA.) with a commercial 3-inch surface coil on anesthetized animals. The T2 measurements used a multislice multi-echo 


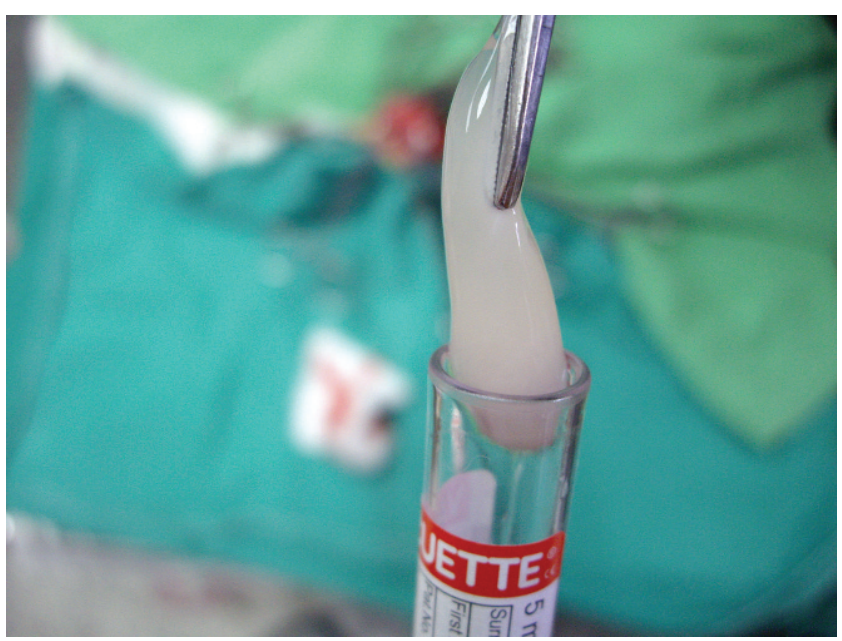

Figure 1 - Platelet-rich fibrin gel obtained from the middle layer of the blood sample after centrifugation.

spin-echo sequence with the following parameters: $\mathrm{TR}=1500 \mathrm{~ms} ; \mathrm{TE}=12,24,36,48 \mathrm{~ms} / 30,60$, 90, and $120 \mathrm{~ms}$; slice thickness $=1.5 \mathrm{~mm}$; matrix size $=256 \times 256$; in-plane resolution $=0.47 \times 0.47 \mathrm{~mm}$; and field of view $=120 \mathrm{~mm}$. The region of interest was manually drawn on the first echo image $(\mathrm{TE}=12 \mathrm{~ms}$ ) to calculate the mean and standard deviation of the $\mathrm{T} 2$ relaxation rate of the defective cartilage.
The femoral cartilage was covered by approximately 2 pixels, and the selected image was enlarged threefold to minimize partial volume effects. The T2 value for each rabbit in group A and group B was compared with that of the same rabbit's normal cartilage on the contralateral side. For comparison under the same conditions, normalized T2 values were calculated using the values for defective cartilage divided by the values for the normal cartilage of the same animal. We used the Mann-Whitney test to compare the normalized T2 values between groups with $p<0.05$ indicating significance.

After sacrificing the animal, all experimental limbs were dissected. The specimens were embedded in paraffin, sectioned, and processed for routine hematoxylin-eosin (HE) staining, which is recommended by the ICRS to assess the degree of cartilage regeneration. ${ }^{8}$

All histology specimens were evaluated by two independent and blinded observers who were experienced in skeletal histopathology. The observers reached a consensus for all samples. Sections of regenerated tissue were graded based on the ICRS Visual Histological Assessment Scale (distribution of cells, mineralization of cartilage, scores for surface and matrix, cell population viability, and subchondral bone abnormalities). ${ }^{9}$ The center of the original defect was used for interpretation following the recommendations of the ICRS. ${ }^{9}$

We used the Kruskal-Wallis test to compare the sums of the ICRS scores among the groups. A nonparametric MannWhitney $U$ test was performed to compare the two groups

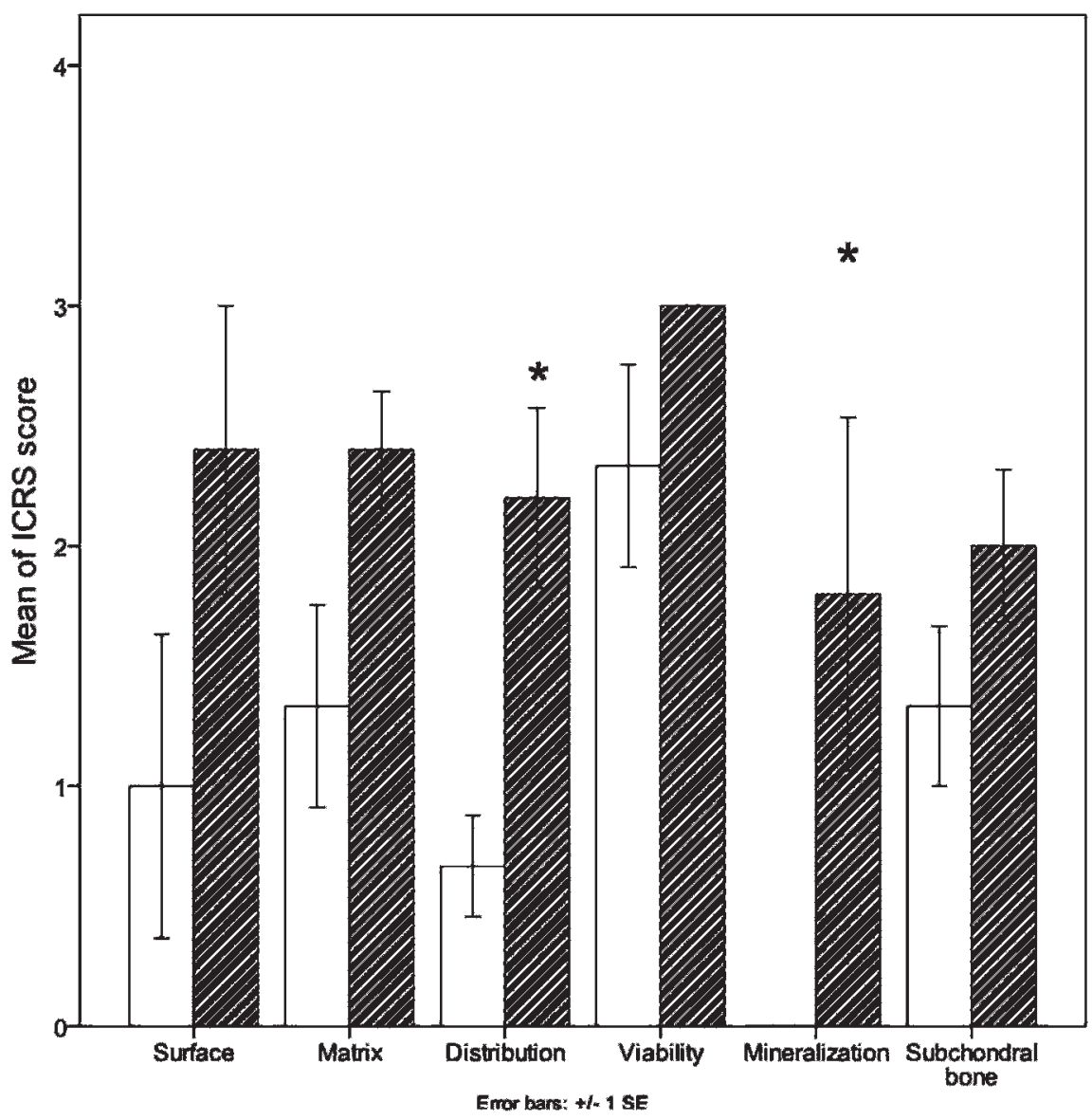

group

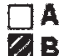

Figure 2 - Bar graph illustrating the International Cartilage Repair Society (ICRS) scores. Significant differences in cell distribution and cartilage mineralization were detected between the experimental group and the control group. * Significant differences. 


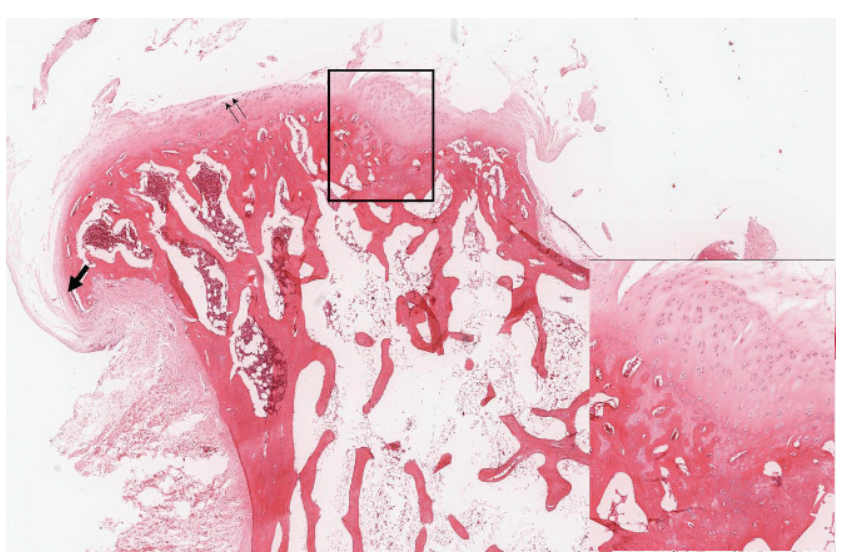

Figure 3 - Group A specimen. Image shows focal thickening of the articular cartilage and the cortical bone (insert) with a deformed articular surface. Fissuring (double arrows) and osteophytes (arrow) are shown (H \& E stain).

with $p<0.05$ indicating a significant difference observed between the groups in the values obtained for the individual parameters.

\section{RESULTS}

The sums of the ICRS scores were significantly different between the groups $(p=0.02)$. Further comparisons showed a significant difference in the cell distribution $(p=0.01)$ and cartilage mineralization $(p=0.03)$ between the groups (Figure 2 ).

In group A (Figure 3), disorganized cell distribution was noted in six specimens. All of the rabbits showed abnormal cartilage mineralization. The cartilage from four of the six rabbits showed surface irregularities and erosion. Two lesions were covered by dense fibrous tissue. In all of the animals, cells had clearly delineated nuclei indicating partial $(2 / 6)$ or predominant viability $(4 / 6)$. We observed subchondral granulation in three animals, detachment at the base in three animals, and marginal osteophytes in three animals.

Specimens from one rabbit in group B were excluded because of a technical failure during tissue preparation. In group B (Figure 4), only one out of the five remaining specimens showed disorganized cell distribution. Three specimens exhibited normal cartilage mineralization. Four of the five specimens showed a smooth and continuous regenerated cartilage surface, and one specimen had major fissures. All five specimens showed hyaline cartilage repair with higher structural integrity of the matrix. The cells were predominantly viable in all five rabbits. Subchondral normal or remodeled tissue was noted in four specimens. No osteophyte formation was noted.

By comparing the means ( \pm standard deviation) and ratios of the MRI T2 values, we observed that the value for the defective cartilage in group A $(11.8 \pm 7.4 \mathrm{~ms}$; ratio: $0.5 \pm 0.1$ ) was lower than that of the normal cartilage of the same rabbit $(23.2 \pm 1.7 \mathrm{~ms}$; ratio: $0.9 \pm 0.2) \quad(p=0.06)$. No differences were detected in the T2 values for the regenerated cartilage in group $\mathrm{B}(18.2 \pm 4.5 \mathrm{~ms}$; ratio: $0.6 \pm 0.2)$ and normal cartilage of the same animal $(p=0.15)$.

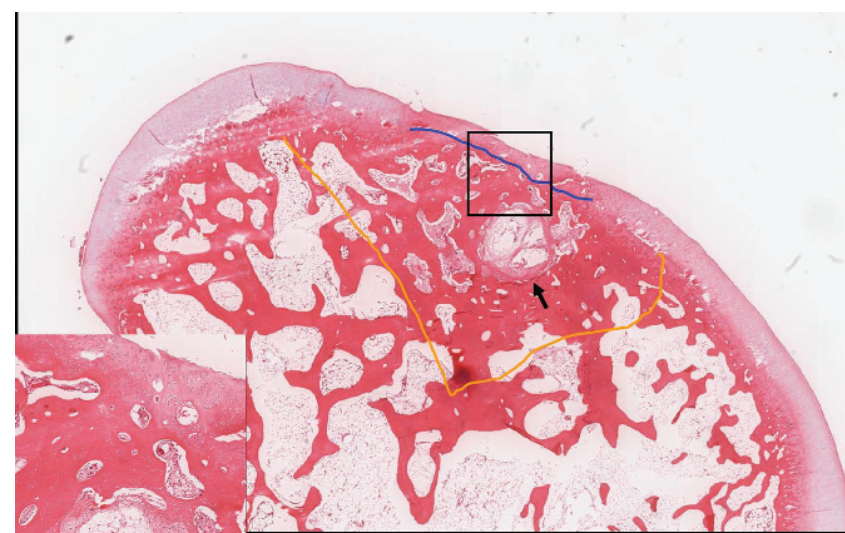

Figure 4 - Group B specimen. Image shows a continuous surface and erosion of the regenerated hyaline cartilage (insert) in the central zone of the original defect area (area within the orange line). A cystic space (arrow) that was filled with granulation tissue in the subchondral area (blue line) with sclerosing change is shown (H \& E stain).

\section{DISCUSSION}

Fibrocartilage contains a high proportion of type I collagen and forms coarse fibers.9 ${ }^{9}$ Fibrocartilage occurs frequently in areas of failed cartilage regeneration, ${ }^{10}$ as observed in our control animals. Mature hyaline cartilage is recognized by the abundant extracellular matrix, which is usually homogeneous, and by the scarcity of rounded chondrocytes that occupy well-defined lacunae, which we observed in our experimental animals.

PRF contains many kinds of growth factors and cytokines. ${ }^{7}$ The growth factors are known to promote cell proliferation, cell differentiation, cell motility, and matrix synthesis by binding to specific cell-surface receptors. ${ }^{11}$ PRF slowly polymerizes during centrifugation. Its connected junctions allow the establishment of a fine and flexible fibrin network that supports cytokine enmeshment, cellular migration, and stem cell trapping. ${ }^{6}$ The cytokines may comprise interleukins, which are needed for inflammatory responses and repair responses to promote normal immune function. Biodegradable artificial scaffolds that are seeded with chondrocytes significantly improve the healing of cartilage defects. The scaffolds improve the redifferentiation capacity of chondrocytes by providing them with appropriate mechanical stability. ${ }^{12}$ We hypothesize that PRF that is mixed with cartilage granules provides favorable conditions for cell migration and cell growth.

Alterations in the MRI T2 values in cartilage correlate with changes in the water content and the organization of collagen structures that are associated with hyaline articular cartilage degradation. $^{13,14}$ In our control animals, the defective cartilage matrix became more fibrotic, which reduced the $\mathrm{T} 2$ value. The regenerated cartilage of treated animals had higher T2 values than did defective cartilage, indicating a decrease in proteoglycans and a progressive increase in collagen content.

Our study indicates that the injured cartilage in rabbits healed within three months. The use of PRF and cartilage granule without bovine thrombin represents a one-step cartilage repair surgery with potentially favorable results. 


\section{ACKNOWLEDGMENTS}

The authors wish to thank Wan Fang Hospital at Taipei Medical University (Key Research Grant 99wf-eva-05) for supporting this project.

\section{AUTHOR CONTRIBUTIONS}

T-F Kuo conceived and designed the study, prepared the animals for surgery and histology, and was responsible for the collection, analysis and interpretation of data. M-F Lin conceived and designed the study, was responsible for the magnetic resonance images, and collection, analysis and interpretation of data. WP Chan conceived and designed the study, was responsible for the critical revision for important intellectual content and for the draft of the manuscript. R-J Su prepared the animals for surgery and histology, and collected the data. Y-H Lin was responsible for the analysis and interpretation of data. $\mathrm{H}-\mathrm{W}$-Lin was responsible for the statistical analysis. Y-C Lin was responsible for the literature revision and for the draft of the manuscript. All the authors have given approval of the version to be published. T-F Kuo and M-F Lin contributed equally to this work and both acted as first author.

\section{REFERENCES}

1. Grande DA, Pitman MI, Peterson L, Menche D, Klein M. The repair of experimentally produced defects in rabbit articular cartilage by autologous chondrocyte transplantation. J Orthop Res. 1989;7:208-18, doi: 10.1002/jor.1100070208.

2. Brittberg M, Lindahl A, Nilsson A, Ohlsson C, Isaksson O, Peterson L. Treatment of deep cartilage defects in the knee with autologous chondrocyte transplantation. N Engl J Med. 1994;331:889-95, doi: 10. 1056/NEJM199410063311401.

3. Hendriks JAA, Adesida AB. Isolation of cells. US Patent Application 20100144036.

4. Murray TG, Parker RD. Restoring cartilage defects: microfracture to autologous chondrocyte implantation using investigational 3D scaffold. Orthopedics. 2007;30:766-7.
5. Simonpieri A, Choukroun J, Girard MO, Ouaknine T, Dohan D. Immediate post-extraction implantation: interest of the PRF. Implantodontie. 2004;13:177-89, doi: 10.1016/j.implan.2004.06.004

6. Choukroun J, Diss A, Simonpieri A, Girard MO, Schoeffler C, Dohan SL, et al. Platelet-rich fibrin (PRF): a second-generation platelet concentrate. Part IV: clinical effects on tissue healing. Oral Surg Oral Med Oral Pathol Oral Radiol Endod. 2006;101:e56-60, doi: 10.1016/j.tripleo.2005.07.011.

7. Dohan DM, Choukroun J, Diss A, Dohan SL, Dohan AJ, Mouhyi J, et al Platelet-rich fibrin (PRF): a second-generation platelet concentrate. Part I: technological concepts and evolution. Oral Surg Oral Med Oral Pathol Oral Radiol Endod. 2006;101:e37-44, doi: 10.1016/j.tripleo.2005.07.008.

8. Mainil-Varlet P, Van Damme B, Nesic D, Knutsen G, Kandel R, Roberts $\mathrm{S}$. A new histology scoring system for the assessment of the quality of human cartilage repair: ICRS II. Am J Sports Med. 2010;38:880-90, doi: $10.1177 / 0363546509359068$

9. Mainil-Varlet P, Aigner T, Brittberg M, Bullough P, Hollander A Hunziker E, et al. International Cartilage Repair Society. Histological assessment of cartilage repair: a report by the Histology Endpoint Committee of the International Cartilage Repair Society (ICRS). J Bone Joint Surg Am. 2003;85-A Suppl 2:45-57.

10. Chiang H, Kuo TF, Tsai CC, Lin MC, She BR, Huang YY, et al. Repair of porcine articular cartilage defect with autologous chondrocyte transplantation. J Orthop Res. 2005;23:584-93, doi: 10.1016/j.orthres.2004.11.003.

11. Lee HJ, Choi BH, Jung JH, Zhu SJ, Lee SH, Huh JY, et al. Maxillary sinus floor augmentation using autogenous bone grafts and platelet-enriched fibrin glue with simultaneous implant placement. Oral Surg Oral Med Oral Pathol Oral Radiol Endod. 2007;103:329-33, doi: 10.1016/j.tripleo. 2006.03.010.

12. Filová E, Jelínek F, Handl M, Lytvynets A, Rampichová M, Varga F, et al. Novel composite hyaluronan/type I collagen/fibrin scaffold enhances repair of osteochondral defect in rabbit knee. J Biomed Mater Res B Appl Biomater. 2008;87:415-24.

13. White LM, Sussman MS, Hurtig M, Probyn L, Tomlinson G, Kandel R Cartilage T2 assessment: differentiation of normal hyaline cartilage and reparative tissue after arthroscopic cartilage repair in equine subjects. Radiology. 2006;241:407-14, doi: 10.1148/radiol.2412051750.

14. Chou MC, Tsai PH, Huang GS, Lee HS, Lee $\mathrm{CH}$, Lin $\mathrm{MH}$, et al. Correlation between the MR T2 value at $4.7 \mathrm{~T}$ and relative water content in articular cartilage in experimental osteoarthritis induced by ACL transection. Osteoarthritis Cartilage. 2009;17:441-7, doi: 10.1016/j.joca. 2008.09.009. 